\title{
The Complexity of Computing a Nash Equilibrium
}

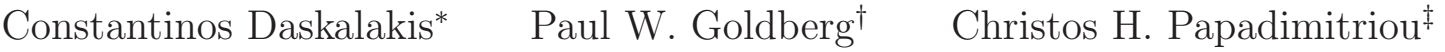

September 26, 2005

\begin{abstract}
We resolve the question of the complexity of Nash equilibrium by showing that the problem of computing a Nash equilibrium in a game with 4 or more players is complete for the complexity class PPAD. Our proof uses ideas from the recently-established equivalence between polynomialtime solvability of normal-form games and graphical games, and shows that these kinds of games can implement arbitrary members of a PPAD-complete class of Brouwer functions.
\end{abstract}

\section{Introduction}

In 1951 Nash showed that every game has a Nash equilibrium (13). The computational problem of finding such equilibria in polynomial time has remained open, and has come under increased scrutiny during the past two decades, see Section 2.2. The 2-player case seems a little easier, since linear programming-like techniques come into play and the solutions are guaranteed to be rational Nash showed in his original paper that there are 3-player games with only irrational equilibria. The problem was known to belong to the class PPAD (17) of search problems with solutions guaranteed to exist by dint of a directed graph-theoretic argument but, unlike the problem of finding a Brouwer fixpoint, it was not known to be complete for that class.

In this paper we show that the problem of computing Nash equilibria in games with 4 players is indeed PPAD-complete. Thus, a polynomial-time algorithm would imply a similar algorithm, e.g., for computing Brouwer fixpoints, a problem for which quite strong lower bounds for large classes of algorithms are known (8), and would have to fail to relativize with respect to the oracles in (1), for which PPAD has no polynomial-time algorithms.

Nash showed his result by reducing the existence of Nash equilibria to the existence of Brouwer fixpoints. Given any game, he constructs a Brouwer function whose fixpoints are precisely the equilibria of the game. In Nash's reduction, as well as in subsequent ones (5), the constructed Brouwer function is quite specialized, and this has led to speculation on whether the fixpoints of such functions (and thus Nash equilibria) are easier to find than for general Brouwer functions. We answer this question in the negative by presenting a reduction in the opposite direction: Any (computationally presented) Brouwer function can be simulated by a game, so that Nash equilibria correspond to fixpoints.

\footnotetext{
${ }^{*}$ University of California at Berkeley, Computer Science Division, Soda Hall, Berkeley, CA 94720, USA. Research supported by NSF ITR grant and a grant from Microsoft Research. email: costis@cs.berkeley.edu

${ }^{\dagger}$ Department of Computer Science, University of Warwick, Coventry CV4 7AL, U.K. Research supported by the EPSRC grant GR/T07343/01 "Algorithmics of Network-sharing Games". This work was begun while the author was visiting UC Berkeley. email: pwg@dcs.warwick.ac.uk

${ }^{\ddagger}$ University of California at Berkeley, Computer Science Division, Soda Hall, Berkeley, CA 94720, USA. Research supported by NSF ITR grant and a grant from Microsoft Research. email: christos@cs.berkeley.edu
} 
In a recent precursor of this paper (7), we describe certain reductions between equilibrium problems; in particular, finding a Nash equilibrium in an $r$-player game can be reduced to the same problem with 4 players, and to that in a graphical game (see (10), a game in which players are nodes on a graph and they affect the utilities of only their neighbors in the graph) with two strategies per player and maximum degree three. Thus, all these problems and their generalizations are PPAD-complete. Our present proof relies crucially on certain "arithmetical gadgets" invented in (7): games that have the effect of adding, multiplying, and comparing real numbers.

The idea in our proof is this: Given a Brouwer function on the 3-dimensional cube (appropriately stylized and presented as a circuit), we simulate the coordinates by three players; each player has two strategies, so that a mixed strategy is essentially a real in $[0,1]$. We construct a game in which the best responses of these three players implement the Brouwer function. This is done by decoding the coordinates into a bit vector, using the circuit to compute the value of the function, and then inducing the three players to add the appropriate increments to their mixed strategy. All this is done by a graphical game, ultimately relying on the reduction in (7) to get to a 4-player normal form games.

There are many difficulties, of course. One has to do with brittle comparators. Our comparator gadget sets a number $z$ to 0 if $x<y$, to 1 if $x>y$, and to anything if $x=y$ (it is not hard to see that no "robust" comparator gadget is possible). This means that decoding is flakey at the boundaries, and at these points the best response can be essentially arbitrary. We solve this by defining our best response to be an average over a "microlattice" around the point of interest, thus smoothing out any effects from boundaries of measure zero. Another problem is that the Brouwer problem, as defined in (17), would be very cumbersome to use in this reduction; thus, a side-product of our proof is a much cleaner canonical version of the computational problem associated with Brouwer's Fixpoint Theorem (actually, one that is, in a sense, half-way between that and Sperner's Lemma).

\section{Definitions, Background, and Preliminaries}

\subsection{Games}

A game in normal form has $r \geq 2$ players (indexed by $p$ ) and for each player $p$ a finite set $S_{p}$ of pure strategies. The set $S$ of pure strategy profiles is the Cartesian products of the $S_{p}$ 's. We denote the set of all strategy profiles of players other than $p$ by $S_{-p}$. Finally, for each $p \leq r$ and $s \in S$ we have a payoff or utility $u_{s}^{p}$ - also occasionally denoted $u_{j s}^{p}$ for $j \in S_{p}$ and $s \in S_{-p}$.

A mixed strategy for player $p$ is a distribution on $S_{p}$, that is, real numbers $x_{j} \geq 0$ for each strategy $j \in S_{p}$ such that $\sum_{j \in S_{p}} x_{j}=1$. A set of $r$ mixed strategies $x_{j}^{p}, p=1, \ldots, r, j \in S_{p}$ are called a (mixed) Nash equilibrium if, for each $p, \sum_{s \in S} u_{s}^{p} x_{s}$ is maximized over all mixed strategies of $p$ (where for a strategy profile $s=\left(s_{1}, \ldots, s_{r}\right) \in S$, we denote by $x_{s}$ the product $x_{s_{1}}^{1} \cdot x_{s_{2}}^{2} \cdots x_{s_{r}}^{r}$ ). That is, a Nash equilibrium is a set of mixed strategies from which no player has an incentive to deviate. It is well-known (see, e.g., (15)) that the following is an equivalent condition for a set of mixed strategies to be a Nash equilibrium:

$$
\sum_{s \in S_{-p}} u_{j s}^{p} x_{s}>\sum_{s \in S_{-p}} u_{j^{\prime} s}^{p} x_{s} \Longrightarrow x_{j^{\prime}}^{p}=0
$$

More generally, a set of mixed strategies is an $\epsilon$-Nash equilibrium for some $\epsilon>0$ if the following holds:

$$
\sum_{s \in S_{-p}} u_{j s}^{p} x_{s}>\sum_{s \in S_{-p}} u_{j^{\prime} s}^{p} x_{s}+\epsilon \Longrightarrow x_{j^{\prime}}^{p}=0
$$


A game in normal form requires $r|S|$ numbers for its description. A graphical game (10) is defined in terms of an undirected graph $G=(V, E)$ together with a set of strategies $S_{v}$ for each $v \in V$. We denote by $\mathcal{N}(v)$ the set consisting of $v$ and $v$ 's neighbors in $G$, and by $S_{\mathcal{N}(v)}$ the set of all $|\mathcal{N}(v)|$-tuples of strategies, one from each vertex in $\mathcal{N}(v)$. In a graphical game, the utilities to $v$ are given by $\left\{u_{s}^{v}: s \in S_{\mathcal{N}(v)}\right\}$. In other words, a graphical game is a succinct representation of a many-player game, when it so happens that for every $p, u_{s}^{p}$ only depends on a few other players.

\subsection{Background Work}

Hitherto there are very few results either positive or negative, for the problem of computing unrestricted Nash equilibria in polynomial time. Lipton and Markakis (11) study the algebraic properties of Nash equilibria, and point out that standard quantifier elimination algorithms can be used to solve them, but these are not polynomial-time in general. The two-player case 2-NASH is the most prominent open problem; the most popular algorithm for 2-Nash is the Lemke-Howson algorithm, shown to be exponential-time in the worst case by Savani and von Stengel (19). For finding specific Nash equilibria, for example the Nash equilibrium with best social cost, the problem is typically NP-complete $(6 ; 3)$.

In addition to $(7)$, the papers $(2 ; 18)$ have started to explore the computational complexity of games, via reductions between alternative types of game.

\section{PPAD}

We now review several definitions and results from the complexity theory of total functions, see (17) for a very similar, but not identical, formalism. A search problem $\mathcal{S}$ is a set of inputs $I_{\mathcal{S}} \subseteq \Sigma^{*}$ such that for each $x \in I_{\mathcal{S}}$ there is an associated set of solutions $\mathcal{S}_{x} \subseteq \Sigma^{|x|^{k}}$ for some integer $k$, such that for each $x \in I_{\mathcal{S}}$ and $y \in \Sigma^{|x|^{k}}$ whether $y \in \mathcal{S}_{x}$ is decidable in polynomial time (notice that this is precisely NP with an added emphasis on finding a witness).

For example, $r$-NASH is the search problem $\mathcal{S}$ in which each $x \in I_{\mathcal{S}}$ is an $r$-player game in normal form together with a binary integer $A$ (the accuracy specification), and $\mathcal{S}_{x}$ is the set of $\frac{1}{A}$-Nash equilibria of the game. Similarly, $d$-GRAPHICAL NASH is the search problem with inputs the set of all graphical games with degree at most $d$, plus an accuracy specification, and solutions the corresponding approximate Nash equilibria.

A search problem is total if $\mathcal{S}_{x} \neq \emptyset$ for all $x \in I_{\mathcal{S}}$. For example, Nash's 1951 theorem (13) implies that $r$-NASH is total. Obviously, the same is true of $d$-GRAPHICAL NASH. The set of all total search problems is denoted TFNP.

Since TFNP is a "semantic" class (i.e., it has no generic complete problem), we explore its complexity via its important subclasses: PLS (9), PPP, PPA and PPAD (17). In particular, PPAD is the class of all total search problems reducible to the following:

END OF THE LINE: Given two circuits $S$ and $P$ with $n$ input bits and $n$ output bits, such that $P\left(0^{n}\right)=0^{n} \neq S\left(0^{n}\right)$, find an input $x \in\{0,1\}^{n}$ such that $P(S(x)) \neq x$ or $S(P(x)) \neq x \neq 0^{n}$.

Intuitively, END OF THE LINE creates a directed graph with vertex set $\{0,1\}^{n}$ and an edge from $x$ to $y$ whenever both $y=S(x)$ and $x=P(y)$ ( $S$ and $P$ stand for "successor candidate" and "predecessor candidate"). This graph has indegree and outdegree at most one, and at least one source, namely $0^{n}$, so it must have a sink. We seek either a sink, or a source other than $0^{n}$. Thus, PPAD is the class of all total functions whose totality is proven via the simple combinatorial argument outlined above (PPAD stands for "polynomial parity argument, directed version").

The following is shown in (17) by a reduction to END OF THE LINE via Brouwer's Theorem and Sperner's Lemma: 
Proposition $1 r$-NASH is in PPAD.

A polynomially computable function $f$ is a polynomial-time reduction from total search problem $\mathcal{S}$ to total search problem $\mathcal{T}$ if for every input $x$ of $\mathcal{S} f(x)$ is an input of $\mathcal{T}$, and furthermore there is another polynomial function $g$ such that for every $y \in \mathcal{T}_{f(x)}, g(y) \in \mathcal{S}_{x}$. In $(7)$ the following is shown:

Theorem 1 There are polynomial-time reductions from $r$-NASH and $d$-GRAPHICAL NASH, for any $r, d \geq 2$, to both 4 -NASH and 3-GRAPHICAL NASH.

A search problem $\mathcal{S}$ in PPAD is called PPAD-complete if all problems in PPAD reduce to it. Obviously, END OF THE LINE is PPAD-complete; our main result in this paper (Theorem 3) states that so are 4-NASH and 3-GRAPHICAL NASH.

In our proof we use a problem we call 3-DIMENSIONAL BROUWER, which is a very simplified version of a problem shown PPAD-complete in (17). We are given a stylized Brouwer function $\phi$ on the 3-dimensional unit cube, defined in terms of its values at the centers of $2^{3 n}$ cubelets with side $2^{-n}$. At the center $c_{i j k}$ of the cubelet $K_{i j k}$ defined as

$$
K_{i j k}=\left\{(x, y, z): i 2^{-n} \leq x \leq(i+1) 2^{-n}, j 2^{-n} \leq y \leq(j+1) 2^{-n}, k 2^{-n} \leq z \leq(k+1) 2^{-n}\right\},
$$

where $i, j, k$ are integers in $\left[2^{n}\right]$, the value of $\phi\left(c_{i j k}\right)$ is $c_{i j k}+\delta_{i j k}$, where $\delta_{i j k}$ is one of the following four vectors:

- $\delta_{1}=(\alpha, 0,0)$

- $\delta_{2}=(0, \alpha, 0)$

- $\delta_{3}=(0,0, \alpha)$

- $\delta_{0}=(-\alpha,-\alpha,-\alpha)$

Here $\alpha>0$ is much smaller than the cubelet side, say $2^{-2 n}$. In the actual (continuous) Brouwer function given by circuit $C$ (described below), the value of $\phi$ near the boundaries of the cubelets would be determined by interpolation - there are many simple ways to do this, and the precise method is of no importance to our discussion. Thus, to compute $\phi$ at the centers of the cubelet $K_{i j k}$ we only need to know which of the four displacements to add. This is computed by a circuit $C$ (which is the only input to the problem) with $3 n$ input bits and 2 output bits; $C(i, j, k)$ is the index $r$ such that, if $c$ is the center of cubelet $K_{i j k}, \phi(c)=c+\delta_{r} . C$ is such that $C(0, j, k)=1$, $C(i, 0, k)=2, C(i, j, 0)=3$ (with conflicts resolved arbitrarily) and $C\left(2^{n}-1, j, k\right)=C\left(i, 2^{n}-1, k\right)=$ $C\left(i, j, 2^{k}-1\right)=0$, so that the function $\phi$ maps the boundary to the interior of the cube. A vertex of a cubelet is called panchromatic if among the eight cubelets adjacent to it there are four that have all four increments $\delta_{0}, \delta_{1}, \delta_{2}, \delta_{3}$.

3-Dimensional Brouwer is thus the following problem: Given a circuit $C$ as described above, find a panchromatic vertex. The relationship with Brouwer fixpoints (as promised by Brouwer's theorem) is that any natural interpolation rule ensures that fixpoints only ever occur in the vicinity of a panchromatic vertex; elsewhere the displacements cannot cancel each other out.

Theorem 2 3-Dimensional Brouwer is PPAD-complete. 
Proof. The reduction is from END OF THE LINE. Given circuits $S$ and $P$ with $n$ inputs and outputs, as prescribed in this problem, we shall construct an equivalent instance of 3-DIMENSIONAL Brouwer, that is, another circuit $C$ with $3 m=3(n+3)$ inputs and two outputs that computes the color of each cubelet of side $2^{-m}$, that is to say, the index $i$ such that $\delta_{i}$ is the correct increment of the Brouwer function at the center of the cubelet encoded into the $3 \mathrm{~m}$ bits of the input. We shall first describe the Brouwer function $\phi$ explicitly, and then argue that it can be computed by a circuit.

Our description of $\phi$ proceeds as follows: We shall first describe a 1-dimensional subset $L$ of the 3 -dimensional unit cube, intuitively an embedding of the path-like directed graph $G_{S, P}$ implicitly given by $S$ and $P$. Then we shall describe the 4-coloring of the $2^{3 m}$ cubelets based on the description of $L$. Finally, we shall argue that colors are easy to compute locally, and that fixpoints correspond to endpoints other than $0^{n}$ of the line.

We assume that the graph $G_{S, P}$ is such that for each edge $(u, v)$, one of the vertices is even (ends in 0 ) and the other is odd; this is easy to guarantee (we could add a dummy input and output bit to $S$ and $P$ that is always flipped.)

$L$ will be orthonormal, that is, each of its segments will be parallel to one of the axes. Let $u \in\{0,1\}^{n}$ be a vertex of $G_{S, P}$. By $[u]$ we denote the integer between 0 and $2^{n}-1$ whose binary representation is $u$; all coordinates of endpoints of segments are integer multiples of $2^{-m}$, a factor that we omit. Associated with $u$ there are two line segments of length 4 of $L$. The first, called the principal segment of $u$, has endpoints $u_{1}=(8[u]+2,2,2)$ and $u_{1}^{\prime}=(8[u]+6,2,2)$. The other auxiliary segment has endpoints $u_{2}=\left(2,8[u]+2,2^{m}-3\right)$ and $u_{2}^{\prime}=\left(2,8[u]+6,2^{m}-3\right)$. Informally, these segments form two dashed lines that run along two edges of the cube and slightly in its interior (see Figure 1).

Now, for every vertex $u$ of $G_{S, P}$, we connect $u_{1}^{\prime}$ to $u_{2}$ by a line with three straight segments, with joints $u_{3}=(8[u]+6,8[u]+2,2)$ and $u_{4}=\left(8[u]+6,8[u]+2,2^{m}-3\right)$. Finally, if there is an edge $(u, v)$ in $G_{S, P}$, we connect $u_{2}^{\prime}$ to $v_{1}$ by a broken line with breakpoints $u_{5}=\left(8[v]+2,8[u]+6,2^{m}-3\right)$ and $u_{6}=(8[v]+2,8[u]+6,2)$. This completes the description of the line $L$. It is easy to see that $L$ traverses the interior of the cube without ever "nearly crossing itself"; that is, two points $p, p^{\prime}$ of $L$ are closer than $4 \cdot 2^{-m}$ in Euclidean distance only if they are connected by a part of $L$ that has length $8 \cdot 2^{-m}$ or less. (This is important in order for the coloring described below of the cubelets surrounding $L$ to be well-defined.) To check this, just notice that segments of different types (e.g., $\left[u_{3}, u_{4}\right]$ and $\left.\left[v_{2}^{\prime}, v_{5}\right]\right)$ come close only if they share an endpoint; segments on the $z=2$ plane are parallel and at least 4 apart; and segments parallel to the $z$ axis differ by at least 4 in either their $x$ or $y$ coordinates.

We now describe the coloring of the $2^{3 m}$ cubelets by four colors corresponding to the four increments. As required for a 3-DIMENSIONAL BROUWER circuit, the coloring of any cubelet $K_{i j k}$ where any one of $i, j, k$ is $2^{m}-1$ is 0 . Given that, any other cubelet with $i=0$ gets color 1 ; with this fixed, any other cubelet with $j=0$ gets color 2 , while the remaining cubelets with $k=0$ get color 3. Having colored the boundaries, we now have to color the interior cubelets. An interior cubelet is always colored 0 unless one of its vertices is a point of the interior of line $L$, in which case it is colored by one of the three other colors in a manner to be explained shortly. Intuitively, at each point of the line $L$, starting from $(2,2,2)$ (the beginning of the principle segment of the string $u=0^{n}$ ) the line $L$ is "protected" from color 0 from all 4 sides. As a result, the only place where the four colors can meet is vertex $u_{2}^{\prime}$ or $u_{1}, u \neq 0^{n}$, where $u$ is an end of the line...

In particular, near the beginning of $L$ at $(2,2,2)$ the 27 cubelets $K_{i j k}$ with $i, j, k \leq 2$ are colored as shown in Figure 2. From then on, for any length-1 segment of $L$ of the form $\left[(x, y, z),\left(x^{\prime}, y^{\prime}, z^{\prime}\right)\right]$ consider the four cubelets containing this edge. Two of these cubelets are colored 3 , and the other two are colored 1 and 2 , in this order clockwise (from the point of view of an observer at $(x, y, z)$ ) 


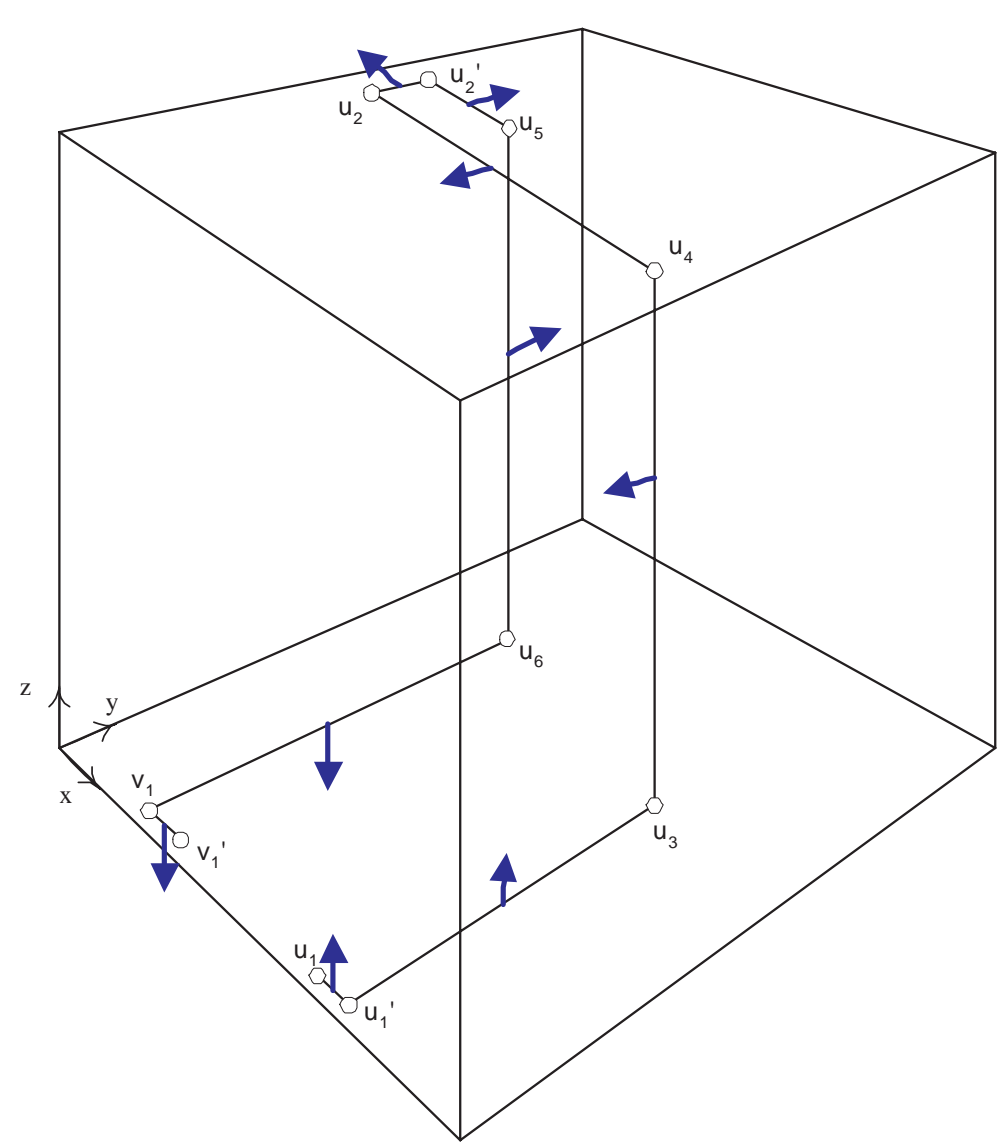

Figure 1: The orthonormal path connecting nodes $(\mathrm{u}, \mathrm{v})$; the arrows indicate the orientation of colors surrounding the path.

as $L$ proceeds from $(2,2,2)$ on. The remaining cubelets touching $L$ are the ones at the joints where $L$ turns. Each of these cubelets, a total of two per turn, takes the color of the two other cubelets adjacent to $L$ with which it shares a face.

Now it only remains to describe, for each line segment $[a, b]$ of $L$, the direction $d$ in which the two cubelets that are colored 3 lie. The rules are these (in Figure 1 the directions $d$ are shown as arrows):

- If $[a, b]=\left[u_{1}, u_{1}^{\prime}\right]$ then $d=(0,0,-1)$ if $u$ is even and $d=(0,0,1)$ if $u$ is odd.

- If $[a, b]=\left[u_{1}^{\prime}, u_{3}\right]$ then $d=(0,0,-1)$ if $u$ is even and $d=(0,0,1)$ if $u$ is odd.

- If $[a, b]=\left[u_{3}, u_{4}\right]$ then $d=(0,1,0)$ if $u$ is even and $d=(0,-1,0)$ if $u$ is odd.

- If $[a, b]=\left[u_{4}, u_{2}\right]$ then $d=(0,1,0)$ if $u$ is even and $d=(0,-1,0)$ if $u$ is odd.

- If $[a, b]=\left[u_{2}, u_{2}^{\prime}\right]$ then $d=(1,0,0)$ if $u$ is even and $d=(-1,0,0)$ if $u$ is odd.

- If $[a, b]=\left[u_{2}^{\prime}, u_{5}\right]$ then $d=(0,-1,0)$ if $u$ is even and $d=(0,1,0)$ if $u$ is odd.

- If $[a, b]=\left[u_{5}, u_{6}\right]$ then $d=(0,-1,0)$ if $u$ is even and $d=(0,1,0)$ if $u$ is odd.

- If $[a, b]=\left[u_{6}, v_{1}\right]$ then $d=(0,0,1)$ if $u$ is even and $d=(0,0,-1)$ if $u$ is odd. 


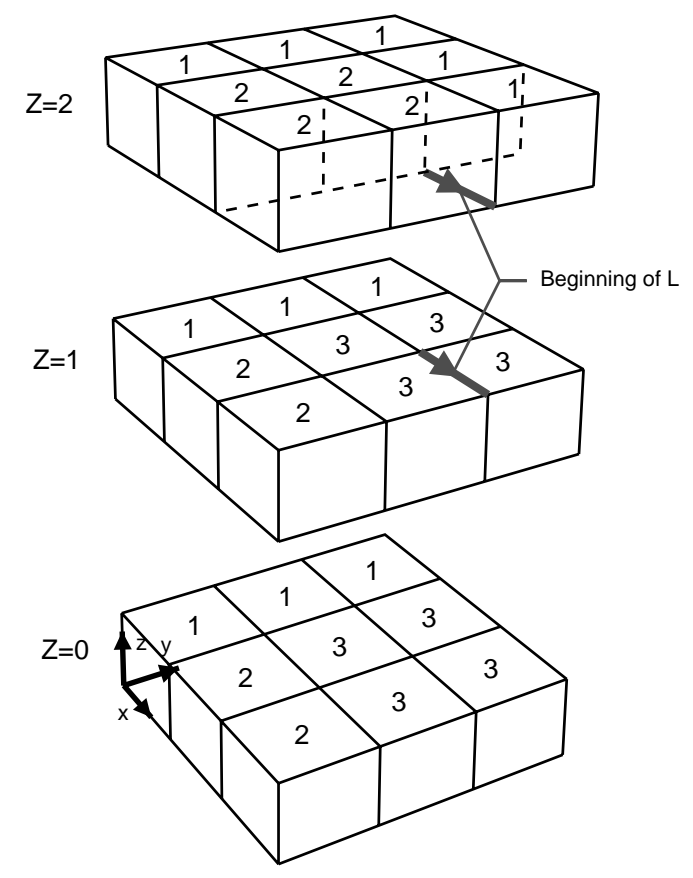

Figure 2: The 27 cubelets around the beginning of line $L$

This completes the description of the construction. Notice that, for this to work, we need our assumption that edges in $G_{S, P}$ go between odd and even nodes. Regarding the alternating orientation of colored cubelets around $L$, note that we could not simply introduce "twists" to make them always point in (say) direction $d=(0,0,-1)$ for all $\left(u_{1}, u_{1}^{\prime}\right)$. That would create a panchromatic cubelet at the location of a twist.

The result now follows from the following two claims:

1. A point in the cube is panchromatic in the described coloring if and only if it is

(a) an endpoint $u_{2}^{\prime}$ of a sink vertex, or

(b) $u_{1}$ of a source vertex $u \neq 0^{n}$ of $G_{S, P}$

2. A circuit $C$ can be constructed in time polynomial in $|S|+|P|$, which computes, for each triple of binary integers $i, j, k<2^{m}$, the color of cubelet $K_{i j k}$.

\section{The Reduction}

We now prove our main result:

Theorem 3 4-NASH is PPAD-complete. 
Proof. The reduction is from 3-Dimensional Brouwer. Given a circuit $C$ with $3 n$ input bits describing a Brouwer function as in the definition of 3-DIMENSIONAL BROUWER, we shall construct a graphical game $\mathcal{G}$, with maximum degree three, that simulates it. Since we know (Theorem 1) that graphical games reduce to $4-\mathrm{NASH}$, this completes the proof.

The graphical game $\mathcal{G}$ is binary in that each vertex $v$ in it has two strategies, and thus, at equilibrium, it represents a real number in $[0,1]$ denoted $\mathbf{p}[v]$. (Letting 0 and 1 denote the strategies, $\mathbf{p}[v]$ is the probability that $v$ plays 1.) There are three distinguished nodes $v_{x}, v_{y}$, and $v_{z}$ representing the coordinates of the Brouwer problem. We next define certain useful gadgets which allow us to make arithmetic operations, variants of the ones described in (7).

Notation: By $x=y \pm \epsilon$ we mean $y-\epsilon \leq x \leq y+\epsilon$.

Lemma 1 There are binary graphical games $\mathcal{G}_{\zeta}$, where $\zeta$ is any real in $[0,1], \mathcal{G}_{=}, \mathcal{G}_{+}, \mathcal{G}_{-}, \mathcal{G}_{*}, \mathcal{G}_{<}$ with four players $a, b, c, d$ such that in all games, the payoffs of a and $b$ do not depend on the choices of the other vertices $c, d$, and

1. at any $\epsilon-N$ ash equilibrium in $\mathcal{G}_{\zeta}$ we have $\mathbf{p}[d]=\zeta \pm \epsilon$;

2. at any $\epsilon$-Nash equilibrium in $\mathcal{G}_{=}$we have $\mathbf{p}[d]=\mathbf{p}[a] \pm \epsilon$;

3. at any $\epsilon$-Nash equilibrium in $\mathcal{G}_{+}$we have $\mathbf{p}[d]=\min \{1, \mathbf{p}[a]+\mathbf{p}[b]\} \pm \epsilon$;

4. at any $\epsilon$-Nash equilibrium in $\mathcal{G}_{-}$in which $\mathbf{p}[a] \geq \mathbf{p}[b]$, we have $\mathbf{p}[d]=\mathbf{p}[a]-\mathbf{p}[b] \pm \epsilon$;

5. at any $\epsilon$-Nash equilibrium in $\mathcal{G}_{*}$ we have $\mathbf{p}[d]=\mathbf{p}[a] \cdot \mathbf{p}[b] \pm \epsilon$;

6. at any $\epsilon$-Nash equilibrium in $\mathcal{G}_{<}$we have $\mathbf{p}[d]=1$ if $\mathbf{p}[a]<\mathbf{p}[b]-\epsilon$ and $\mathbf{p}[d]=0$ if $\mathbf{p}[a]>$ $\mathbf{p}[b]+\epsilon$.

Proof. This is a simple extension of Propositions 1-3 of (7). Player $c$ is needed for $\mathcal{G}_{=}, \mathcal{G}_{+}, \mathcal{G}_{-}$ and $\mathcal{G}_{*}$; its role is to mediate between $a, b$ and $d$.

The constructions are straightforward, for example for item 2 , the payoffs in $\mathcal{G}=$ are

\begin{tabular}{ll|cc} 
& & $c$ plays 0 & $c$ plays 1 \\
\cline { 2 - 4 } Payoffs to $d:$ & $d$ plays 0 & 0 & 1 \\
& $d$ plays 1 & 1 & 0
\end{tabular}

Payoffs to $c$ :

\begin{tabular}{|c|c|c|c|c|c|c|}
\hline \multirow{3}{*}{$c$ plays 0} & & $d$ plays $0 \quad d$ plays 1 & \multirow{3}{*}{$c$ plays 1} & & $d$ plays 0 & $d$ plays 1 \\
\hline & $a$ plays 0 & 0 & & $a$ plays 0 & 0 & 1 \\
\hline & $a$ plays 1 & 1 & & $a$ plays 1 & 0 & 1 \\
\hline
\end{tabular}

If $c$ plays 1 , then the expected payoff to $c$ is $\mathbf{p}[d]$, and if $c$ plays 0 then the expected payoff to $c$ is $\mathbf{p}[a]$. Therefore, in a $\epsilon$-Nash equilibrium of $\mathcal{G}_{=}$, if $\mathbf{p}[d]>\mathbf{p}[a]+\epsilon$ then $\mathbf{p}[c]=1$.

However, note also that if $\mathbf{p}[c]=1$ then $\mathbf{p}[d]=0$. (Payoffs to $d$ make it prefer to disagree with c.) Consequently, $\mathbf{p}[d]$ cannot be strictly larger than $\mathbf{p}[a]+\epsilon$.

Similarly, if $\mathbf{p}[d]<\mathbf{p}[a]-\epsilon$ then $\mathbf{p}[c]=0$, which implies that $\mathbf{p}[d]=1$ (again since $d$ has the biggest payoff by disagreeing with $c$ ). Hence $\mathbf{p}[d]$ cannot be less than $\mathbf{p}[a]-\epsilon$.

For Item 3 , the payoffs in $\mathcal{G}_{+}$are 


\begin{tabular}{ll|cc} 
& & $c$ plays 0 & $c$ plays 1 \\
\cline { 2 - 4 } Payoffs to $d:$ & $d$ plays 0 & 0 & 1 \\
& $d$ plays 1 & 1 & 0
\end{tabular}

\begin{tabular}{|c|c|c|c|c|c|c|c|}
\hline & & & $b$ plays 0 & $b$ plays 1 & & & \\
\hline Payoffs to $c$ : & $c$ plays 0 & $a$ plays 0 & 0 & 1 & $c$ plays 1 & $d$ plays 0 & 0 \\
\hline
\end{tabular}

If $c$ plays 1 , then the expected payoff to $c$ is $\mathbf{p}[d]$, and if $c$ plays 0 then the expected payoff to $c$ is $\mathbf{p}[a]+\mathbf{p}[b]$. Therefore, in a $\epsilon$-Nash equilibrium of $\mathcal{G}_{+}$, if $\mathbf{p}[d]>\mathbf{p}[a]+\mathbf{p}[b]+\epsilon$ then $\mathbf{p}[c]=1$.

However, note from the payoffs to $d$ that if $\mathbf{p}[c]=1$ then $\mathbf{p}[d]=0$. Consequently, $\mathbf{p}[d]$ cannot be strictly larger than $\mathbf{p}[a]+\mathbf{p}[b]+\epsilon$.

Similarly, if $\mathbf{p}[d]<\mathbf{p}[a]+\mathbf{p}[b]-\epsilon$ then due to the payoffs to $c$ we have $\mathbf{p}[c]=0$. This in turn implies that $\mathbf{p}[d]=1$ ( since $d$ has the biggest payoff by disagreeing with $c$ ). Hence $\mathbf{p}[d]$ cannot be less than $\min (1, \mathbf{p}[a]+\mathbf{p}[b]-\epsilon)$.

$\mathcal{G}_{<}$is as follows: player $d$ receives a payoff of 1 if $d$ plays 0 and $a$ plays 1 , and $d$ receives a payoff of 1 if $d$ plays 1 and $b$ plays 1 , otherwise $d$ received a payoff of 0 .

Equivalently, $d$ receives an expected payoff of $\mathbf{p}[a]$ if $d$ plays 0 , and receives $\mathbf{p}[b]$ of $d$ plays 1 . It can be verified that this implements $\mathcal{G}_{<}$.

Notice that, in $\mathcal{G}_{<}, \mathbf{p}[d]$ is arbitrary if $\mathbf{p}[a]$ is close to $\mathbf{p}[b]$, hence we called it the brittle comparator. As an aside, it is not hard to see that a robust comparator, one in which $d$ is guaranteed at a precise Nash equilibrium to be, say, 0 if $\mathbf{p}[a]=\mathbf{p}[b]$, cannot exist, since it could be used to produce a simple graphical game with no Nash equilibrium, contradicting Nash's theorem.

To continue the reduction, the graphical game $\mathcal{G}$ will contain the following vertices.

- the three coordinate vertices $v_{x}, v_{y}, v_{z}$,

- for $i \in[n]$, vertices $v_{b_{i}(x)}, v_{b_{i}(y)}$ and $v_{b_{i}(z)}$, whose $\mathbf{p}$-values represent the $i$-th bit of $\mathbf{p}\left[v_{x}\right], \mathbf{p}\left[v_{y}\right]$, $\mathbf{p}\left[v_{z}\right]$

- $v_{x_{i}}, v_{y_{i}}$ and $v_{z_{i}}$ (for $i \in[n]$ ), vertices whose $\mathbf{p}$-values represent the first $i$ bits of $\mathbf{p}\left[v_{x}\right], \mathbf{p}\left[v_{y}\right]$, $\mathbf{p}\left[v_{z}\right]$

We can extract these values by computing the binary representation of $\left\lfloor\mathbf{p}\left[v_{x}\right] 2^{n}\right\rfloor$ and similarly for $v_{y}$ and $v_{z}$ — that is, the binary representations of the integers $i, j, k$ such that $(x, y, z)=$ $\left(\mathbf{p}\left[v_{x}\right], \mathbf{p}\left[v_{y}\right], \mathbf{p}\left[v_{z}\right]\right)$ lies in the cubelet $K_{i j k}$. This is done by a graphical game that simulates, using the arithmetical gadgets of Lemma 1, the following algorithm (by $<(a, b)$ we mean the brittle comparator of $a$ and $b$ ):

$$
\begin{aligned}
& x_{1}=x\left(\text { short for } \mathbf{p}\left[x_{1}\right]=\mathbf{p}\left[v_{x}\right]\right) \\
& \text { for } i=1, \ldots, n \text { do: } \\
& \left\{b_{i}(x):=<\left(2^{-i}, x_{i}\right) ; x_{i+1}:=x_{i}-b_{i}(x) \cdot 2^{-i}\right\}
\end{aligned}
$$

Similarly for $y$ and $z$.

This is accomplished in $\mathcal{G}$ by connecting these nodes as prescribed by Lemma 1 , so that $\mathbf{p}\left[v_{x_{i}}\right], \mathbf{p}\left[v_{b_{i}(x)}\right]$, etc. approximate the value of $x_{i}, b_{i}(x)$ etc. as computed by the above algorithm. The following lemma (when applied with $m=n$ ) shows that this device properly decodes the first $n$ bits of the binary expansion of $x=\mathbf{p}\left[v_{x}\right]$, as long as $x$ is not too close to a multiple of $2^{-n}$. 
Lemma 2 For $m \leq n$, if $\sum_{i=1}^{m} b_{i}(x) 2^{-i}+2 m \epsilon<x<\sum_{i=1}^{m} b_{i}(x) 2^{-i}+2^{-m}-2 m \epsilon$ for some $b_{1}, \ldots, b_{m} \in\{0,1\}$, then at any $\epsilon-N a s h$ equilibrium of $\mathcal{G}, \mathbf{p}\left[v_{b_{m}(x)}\right]=b_{m}$, and $\mathbf{p}\left[v_{x_{m+1}}\right]=x-$ $\sum_{i=1}^{m} b_{i}(x) 2^{-i} \pm 2 m \epsilon$.

Proof. Induction on $m$. It is trivial for $m=0$, so suppose it holds up to $m$. Then we know that $\mathbf{p}\left[v_{x_{m}}\right]$ is not within $\epsilon$ of a multiple of $2^{-m}$, and therefore $\mathbf{p}\left[v_{b_{m+1}(x)}\right]$ will hold the correct value of the next bit. Finally, the multiplication and subtraction needed to compute $x_{m+1}$ will each introduce an additional error of $\epsilon$, concluding the induction.

The above is repeated for $y$ and $z$ to obtain $i, j, k$, that is, $3 n$ vertices of the graph of $\mathcal{G}$ whose $\mathbf{p}$ values correspond to the bits of $i, j, k$ (assuming that $x, y, z$ are all at least $2 n \epsilon$ away from any multiple of $2^{-n}$ ). Once we have the binary representations of $i, j, k$, we can feed them into another part of $\mathcal{G}$ that simulates the circuit $C$. We could simulate the circuit by having nodes that represent gates, using addition (with ceiling 1 ) to simulate or, multiplication for and, and $1-x$ for negation. However, there is a simpler way, one that avoids the complications related to accuracy, to simulate Boolean functions under the assumption that the inputs are 0 or 1 :

Lemma 3 There are binary graphical games $\mathcal{G}_{\vee}, \mathcal{G}_{\wedge}, \mathcal{G}_{\neg}$ with two input players a, $b$ (one input player a for $\left.\mathcal{G}_{\neg}\right)$ and an output player $c$ such that the payoffs of $a$ and $b$ do not depend on the choices of $c$, and, at any $\epsilon$-Nash equilibrium with $\epsilon<1 / 4$ in which $\mathbf{p}[a], \mathbf{p}[b] \in\{0,1\}, \mathbf{p}[c]$ is also in $\{0,1\}$, and is in fact the result of applying the corresponding Boolean function to the inputs.

Proof. These games are in the same spirit as $G_{<}$. In $G_{\vee}$, for example, the payoff to $c$ is $1 / 2$ if it plays 0; if $c$ plays 1 its payoff is 1 if at least one of $a, b$ plays 1 , and it is 0 if they both play 0 . Similarly for $G_{\wedge}$ and $G_{\neg}$

Thus in addition to the part of $\mathcal{G}$ that computes the bits $\mathbf{p}\left[v_{b_{i}(x)}\right]$ etc. of $i, j, k$ we have a part that simulates $C$, containing one player for each gate of $C$, and such that, in any $\epsilon$-approximate equilibrium in which all the $\mathbf{p}\left[v_{b_{i}(x)}\right]$ 's are $0-1$, the players corresponding to the outputs of $C$ also play pure strategies, and furthermore these strategies correspond correctly to the outputs of $C$.

In fact, it is convenient to assume that the output of $C$ is a little more explicit: $C$ computes six bits $\Delta x^{+}, \Delta x^{-}, \Delta y^{+}, \Delta y^{-}, \Delta z^{+}, \Delta z^{-}$such that at most one of $\Delta x^{+}, \Delta x^{-}$is 1 , and at most one of $\Delta y^{+}, \Delta y^{-}$is 1 , and similarly for $z$, and the increment of the Brouwer function at the center of $K_{i j k}$ is $\alpha \cdot\left(\Delta x^{+}-\Delta x^{-}, \Delta y^{+}-\Delta y^{-}, \Delta z^{+}-\Delta z^{-}\right)$, one of $\delta_{0}, \delta_{1}, \delta_{2}, \delta_{3}$ as defined above. It would seem that all we have to do now is close the loop by incentivizing (using the $G_{+}$device of Lemma 1) the $v_{x}, v_{y}$ and $v_{z}$ vertices to increment their $\mathbf{p}$ values by the appropriate amounts.

But, as we mentioned above, there is a problem: Because of the brittle comparators, at the boundaries of the cubelets the vertices that should represent the values of the bits of $i, j, k$ hold in fact arbitrary reals, and therefore so do the output vertices of $C$, the $\Delta x^{+}$etc, and this noise in the calculation can create spurious Nash equilibria. Suppose for example that $(x, y, z)$ lies on the boundary between two cubelets that have color 1 . Then there ought not to be a Nash equilibrium with $\mathbf{p}\left[v_{x}\right]=x, \mathbf{p}\left[v_{y}\right]=y, \mathbf{p}\left[v_{z}\right]=z$. We want that if $\mathbf{p}\left[v_{x}\right]=x, \mathbf{p}\left[v_{y}\right]=y, \mathbf{p}\left[v_{z}\right]=z$, then $\mathbf{p}\left[v_{x}\right], \mathbf{p}\left[v_{y}\right], \mathbf{p}\left[v_{z}\right]$ should have the incentive to move in direction $\delta_{1}$, so that $v_{x}$ prefers to increase $\mathbf{p}\left[v_{x}\right]$. However, on a boundary between two cubelets, some of the "bit values" that get loaded into $v_{b_{i}(x)}$, could be other than 0 and 1 , and then there is nothing we can say about the output of the circuit that processes these values.

To overcome this difficulty, we resort to the following averaging maneuver: We repeat the above computation not just for the point $(x, y, z)$, but for all $M=(2 m+1)^{3}$ points of the form $(x+p \cdot \alpha, y+q \cdot \alpha, z+s \cdot \alpha)$ for $-m \leq p, q, s \leq m$, where $m$ is a large enough constant to be fixed 
later (we show below that $m=20$ is sufficient). This is done by $M$ copies of the device described above, yielding $6 M$ output bits $\Delta x_{1}^{+}, \ldots, \Delta z_{M}^{-}$. A final part of $\mathcal{G}$ calculates the vector

$$
(\delta x, \delta y, \delta z)=\frac{\alpha}{M} \sum_{t=1}^{M}\left(\Delta x_{t}^{+}-\Delta x_{t}^{-}, \Delta y_{t}^{+}-\Delta y_{t}^{-}, \Delta z_{t}^{+}-\Delta z_{t}^{-}\right),
$$

the average increment of all $M$ points (this is done using the arithmetic gadgets of Lemma 1).

We can now close the loop by inserting addition gadgets that force, at equilibrium, $\mathbf{p}\left[v_{x}\right]$ to be $x+\delta x$, where by $x$ we mean the value of a vertex that is a copy of $v_{x}$, and similarly for $v_{y}$ and $v_{z}$. This concludes the reduction; it is clear that it can be carried out in polynomial time.

Our proof is concluded by the following claim. For the following lemma we choose $\epsilon=\alpha^{2}$. Recall from our definition of 3-Dimensional Brouwer that we chose $\alpha=2^{-2 n}$.

Lemma 4 In any $\epsilon$-Nash equilibrium of the game $\mathcal{G}$, one of the vertices of the cubelet that contains $\left(\mathbf{p}\left[v_{x}\right], \mathbf{p}\left[v_{y}, \mathbf{p}\left[v_{z}\right]\right)\right.$ is panchromatic.

Proof. We start by pointing out a simple property of the increments $\delta_{0}, \ldots, \delta_{3}$ :

Lemma 5 Suppose that for nonnegative integers $k_{0}, \ldots, k_{3}$ all three coordinates of $\sum_{i=0}^{3} k_{i} \delta_{i}$ are smaller in absolute value than $\frac{\alpha K}{5}$ where $K=\sum_{i=0}^{3} k_{i}$. Then all four $k_{i}$ are positive.

Proof. For the sake of contradiction, suppose that $k_{1}=0$. It follows that $k_{0}<K / 5$ (otherwise the negative $x$ component would be too large), and thus one of $k_{2}, k_{3}$ is larger than $2 K / 5$, which makes the corresponding component too large, a contradiction. Similarly if $k_{2}=0$ or $k_{3}=0$. Finally, if $k_{0}=0$ then one of $k_{1}, k_{2}, k_{3}$, and the associated component, is at least $K / 3$, again a contradiction.

The small value of $\epsilon$ relative to $\alpha$ first implies that, for direction $x$, at most one of the values $x+p \cdot \alpha$ can be $2 n \epsilon$-close to a multiple of $2^{-n}$, and similarly for $y$ and $z$. Hence, from among the $M=(2 m+1)^{3}$ evaluations, all but at most $3(2 m+1)^{2}$, or at least $K=(2 m-2)(2 m+1)^{2}$, compute legitimate $\Delta x^{+}$etc. values. These $K$ terms add up to $\frac{1}{M} \sum_{i=0}^{3} k_{i} \delta_{i}$ for some nonnegative integers $k_{0}, \ldots, k_{3}$ adding up to $K$ (recall that from lemma 3 these $K$ evaluations of the circuit will produce binary outputs). The remaining terms can add up to a vector with each coordinate bounded from above in absolute value by $\frac{\alpha}{M} 6(2 m+1)^{2}$. Thus, $\sum_{i=0}^{3} k_{i} \delta_{i}$ must add up to a vector with each coordinate bounded in absolute value by $\alpha 6(2 m+1)^{2}+2 M \epsilon$. By choosing $m=20$, the bound becomes less than $\alpha K / 5$, and so Lemma 5 applies. It follows that among the results of the $K$ computations of the increments, all four $\delta_{0}, \ldots, \delta_{3}$ appeared. This implies that among the corners of the cubelets containing $(x, y, z)$ there must be one panchromatic corner, completing the proof of Lemma 4.

To conclude the proof of Theorem 3, if we find any $\epsilon$-Nash equilibrium of $\mathcal{G}$, Lemma 4 has shown that by reading off the first $n$ binary digits of $\mathbf{p}\left[v_{x}\right], \mathbf{p}\left[v_{y}\right]$ and $\mathbf{p}\left[v_{z}\right]$ we obtain a solution to the corresponding instance of 3-DIMENSIONAL BROUWER.

\section{Open Problems}

Our work leaves open the cases with 2 and 3 players. We conjecture that the 3 -player case is also PPAD-complete (even though considerable more work and new ideas may be required to prove this). In contrast, we believe that there is a polynomial-time algorithm for solving 2-player games. Similarly, we believe that graphical games with maximum degree two should also be solvable. 
Finally, the important problem of computing approximate Nash equilibria with less than exponential accuracy is also left wide open.

\section{References}

[1] P. Beame, S. Cook, J. Edmonds, R. Impagliazzo, T. Pitassi. "The Relative Complexity of NP Search Problems," J. Comput. Syst. Sci. 57, 1, pp. 13-19, 1998.

[2] B. Codenotti, A. Saberi, K. Varadarajan and Y. Ye. "Leontief Economies Encode Nonzero Sum Two-Player Games" Electronic Colloquium in Computational Complexity TR-05-055, 2005. To appear in SODA 2006.

[3] V. Conitzer and T. Sandholm. "Complexity Results about Nash Equilibria," Proceedings of 18th IJCAI, pp. 765-771, Acapulco, Mexico, 2003.

[4] A. Fabrikant, C.H. Papadimitriou and K. Talwar. "The Complexity of Pure Nash Equilibria," Proceedings of 36th STOC pp. 604-612, Chicago, Illinois, USA, Jun 2004.

[5] J. Geanakoplos. "Nash and Walras Equilibrium via Brouwer," Economic Theory, 21, 2003.

[6] I. Gilboa and E. Zemel. "Nash and correlated equilibria: Some complexity considerations," Games and Economic Behavior, 1989.

[7] P. W. Goldberg and C. H. Papadimitriou. "Reducibility Among Equilibrium Problems," Electronic Colloquium in Computational Complexity TR-05-090, 2005.

[8] M. Hirsch, C. H. Papadimitriou and S. Vavasis. "Exponential Lower Bounds for Finding Brouwer Fixpoints," J. Complexity 5, pp. 379-416, 1989.

[9] D. S. Johnson, C. H. Papadimitriou and M. Yannakakis, "How Easy is Local Search?," J. Comput. Syst. Sci. 37, 1, pp. 79-100,1988.

[10] M. Kearns, M. Littman and S. Singh. "Graphical Models for Game Theory," Proceedings of UAI, 2001.

[11] R. Lipton and E. Markakis. "Nash Equilibria via Polynomial Equations," Proceedings of the 6th Latin American Symposium on Theoretical Informatics (LATIN'04), pp. 413-422, Buenos Aires, Argentina, 2004.

[12] M. Littman, M. Kearns and S. Singh. "An Efficient Exact Algorithm for Single Connected Graphical Games," Proceedings of 14 th NIPS (Advances in Neural Information Processing Systems), pp. 817-823, 2002.

[13] J. Nash. "Noncooperative Games," Annals of Mathematics, 54, 289-295, 1951.

[14] J. von Neumann and O. Morgenstern. Theory of Games and Economic Behavior, Princeton University Press, 1944.

[15] M.J. Osborne and A. Rubinstein. A Course in Game Theory, MIT Press (1994).

[16] C. H. Papadimitriou. "Computing Correlated Equilibria in Multiplayer Games," Proceedings of STOC, 2005. 
[17] C. H. Papadimitriou. "On the Complexity of the Parity Argument and Other Inefficient Proofs of Existence," J. Comput. Syst. Sci. 48, 3, pp. 498-532, 1994.

[18] G. Schoenebeck and S. Vadhan. "The Computational Complexity of Nash Equilibria in Concisely Represented Games," Electronic Colloquium in Computational Complexity TR-05-052, 2005.

[19] R. Savani and B. von Stengel. "Exponentially many steps for finding a Nash equilibrium in a Bimatrix Game". Proceedings of 45th FOCS, pp. 258-267, 2004. 